\title{
Evaluation of deformable image registration for contour propagation between CT and cone-beam CT images in adaptive head and neck radiotherapy
}

\author{
X. Li ${ }^{\mathrm{a}}$, Y.Y. Zhang ${ }^{\mathrm{b}}$, Y.H. Shi ${ }^{\mathrm{b}}$, L.H. Zhou ${ }^{\mathrm{a}, *}$ and X. Zhen ${ }^{\mathrm{a}, *}$ \\ ${ }^{a}$ Department of Biomedical Engineering, Southern Medical University, Guangzhou, Guangdong, China \\ ${ }^{\mathrm{b}}$ Department of Radiotherapy Oncology, the First Hospital of Jilin University, Changchun, Jilin, China
}

\begin{abstract}
Deformable image registration (DIR) is a critical technic in adaptive radiotherapy (ART) to propagate contours between planning computerized tomography (CT) images and treatment CT/Cone-beam CT (CBCT) image to account for organ deformation for treatment re-planning. To validate the ability and accuracy of DIR algorithms in organ at risk (OAR) contours mapping, seven intensity-based DIR strategies are tested on the planning CT and weekly CBCT images from six Head $\&$ Neck cancer patients who underwent a $6 \sim 7$ weeks intensity-modulated radiation therapy (IMRT). Three similarity metrics, i.e. the Dice similarity coefficient (DSC), the percentage error (PE) and the Hausdorff distance (HD), are employed to measure the agreement between the propagated contours and the physician delineated ground truths. It is found that the performance of all the evaluated DIR algorithms declines as the treatment proceeds. No statistically significant performance difference is observed between different DIR algorithms ( $p>0.05$ ), except for the double force demons (DFD) which yields the worst result in terms of DSC and PE. For the metric HD, all the DIR algorithms behaved unsatisfactorily with no statistically significant performance difference $(p=0.273)$. These findings suggested that special care should be taken when utilizing the intensitybased DIR algorithms involved in this study to deform OAR contours between CT and CBCT, especially for those organs with low contrast.
\end{abstract}

Keywords: Adaptive radiotherapy, deformable image registration, CBCT, contour propagation, head \& neck cancer

\section{Introduction}

The intensity-modulated radiation therapy (IMRT) plays a critical role in the management of head and neck $(\mathrm{H} \& \mathrm{~N})$ cancer patients [1]. IMRT can maximize tumor coverage and sparing of organs at risk (OARs) by generating steep dose gradient, and thus leads to a potential increase in the therapeutic outcome [2]. However, it does not take into account of fractional anatomical changes, such as tumor shrinkage, nodal/glandular volume, weight loss and geometric variations, etc., during a typical $5 \sim 7$ week treatment course [3]. The adaptive radiation therapy (ART) [4] is a possible solution to overcome these limitations by adapting temporal changes in anatomy with daily imaging in the radiotherapy process [5].

\footnotetext{
${ }^{*}$ Corresponding authors: L.H. Zhou, X. Zhen, Department of Biomedical Engineering, Southern Medical University, Guangzhou, Guangdong 510515, China. E-mail: smart@smu.edu.cn (LZ), xinzhen@ smu.edu.cn (XZ).
}

0928-7329/16/\$35.00 @ 2016 - IOS Press and the authors. All rights reserved This article is published online with Open Access and distributed under the terms of the Creative Commons Attribution NonCommercial License. 
In a typical online ART process, a cone-beam computed tomography (CBCT) scan is usually performed to obtain three-dimensional patient information prior to treatment. Then, deformable image registration (DIR) technique is utilized to establish the point-point correspondence between the planning computed tomography (CT) and the CBCT. The resulting deformation vector field (DVF) can be used for (1) propagating contoured region of interest (ROI) from one image to another [6] and (2) deformation of the re-planned dose maps to integrate the accumulated dose given to the patient [7].

Effective and reliable ART relies upon accurate DIR-propagated ROIs, and thus it is necessary to verify the accuracy of the available DIR algorithms for use in a clinical setting. There is a variety of studies assessing the quality of CT-CT DIR algorithms in $\mathrm{H} \& \mathrm{~N}$ cancer patients, for instance, Castadot et al. [8] compared twelve voxel-based DIR strategies in ROI propagation performance using expert physician drawn contours as benchmarks. Hardcastle et al. [9] investigated the clinical utility of two DIR algorithms for ROIs propagation using CT image data from five institutions. They reported good anatomical agreements for OARs, but claimed that the propagated target structures need to be thoroughly reviewed before clinically use. Mohamed et al. [10] developed a quality assurance workflow for quantitative assessment of four different DIR technics used for $\mathrm{H} \& \mathrm{~N}$ radiation therapy-simulation $\mathrm{CT}$ with diagnostic CT coregistration. There are scarcer studies, however, focused on the CT-CBCT DIR algorithm comparison with $\mathrm{H} \& \mathrm{~N}$ patient data. Though a number of CT-CBCT DIR methods for different clinical sites have been developed [11-14], it is acknowledged that some DIR algorithms may be more suitable for specific anatomies and image modalities due to the mathematical basis of the algorithms [15]. In this sense, it is beneficial to assess the clinical acceptability of the available DIR algorithms to reduce the time and resources required for contour reviewing and correction in the CBCT-based ART process [9].

The aim of this study is to develop a strict and objective methodology to compare different DIR strategies for the purpose of contour propagation from the planning CT to the treatment CBCT of H \& $\mathrm{N}$ cancer patient in the adaptive radiotherapy setting.

\section{Materials and methods}

\subsection{Clinical data}

Clinical data from six nasopharyngeal cancer (NPC) patients were retrospectively collected for DIR performance evaluations in this study. The characteristics of all patients were listed in Table 1. All patients were treated by IMRT with weekly CBCT for setup error correction. Patient immobilization during the acquisition of planning CT (named $\mathrm{pCT}$ ) and $\mathrm{CBCT}$ was achieved by a customized thermoplastic mask. All the pCT images in the treatment position were reconstructed with a voxel size of $1.60 \times 1.60$ $\times 5 \mathrm{~mm}^{3}$. Weekly kilovoltage CBCT with a resolution of $0.51 \times 0.51 \times 1.99 \mathrm{~mm}^{3}$ was acquired just before the treatment delivery. Rigid registration is performed before applying the DIR, and the transversal size for all the $\mathrm{pCT}$ and $\mathrm{CBCT}$ images were $512 \times 512$. A total number of six pCT images and thirty-one $\mathrm{CBCT}$ images were involved in this study.

\subsection{Regions of interest delineation}

Two organs at risk (OARs), i.e. the parotid glands (PG) and the submandibular glands (SMG), on both the pCT and weekly CBCT were manually delineated by the same radiation oncologist on a commercial treatment planning system (Eclipse 10.0, Varian), to validate the agreement between the deformed and reference OARs. We consider the left and right part of the PGs and SMGs as individual organs in organ 
Table 1

Characteristics of six patients

\begin{tabular}{cccccc}
\hline Patients & Sex & Age (years) & Stages & Histology & Concurrent CBCT \\
\hline 1 & F & 44 & T4N1M0 & NPC & Yes \\
2 & F & 48 & T3N3M0 & NPC & Yes \\
3 & F & 59 & T2N2M0 & NPC & Yes \\
4 & M & 64 & T3N1M0 & NPC & Yes \\
5 & M & 39 & T3N2M0 & NPC & Yes \\
6 & M & 40 & T4N1M0 & NPC & Yes \\
\hline
\end{tabular}

deformation, thus, the PGs is separated into 1PG (left) and rPG (right), and similarly, 1SMG (left) and rSMG (right) for the SMGs. Note that the clinical target volumes (CTVs) or gross target volumes (GTVs) were not used in this study mainly because it is challenging to identify the contrast-lack CTVs/GTVs on the CBCT images, and inaccurate organ delineations will potentially bias the results. Therefore, we focus our attentions on the above two OARs, which have been studied extensively and are the first priority for radiation complication control since they might shrink and deform evidently inward to the higher dose region during the $H \& N$ radiation therapy.

\subsection{Data preprocessing}

All the $\mathrm{CT}$ and $\mathrm{CBCT}$ images were pre-processed before performing the registration. The treatment table and thermoplastic mask were removed using the free, open source software 3D Slicer (version 4.3.1, http://www.slicer.org) [16], leaving purely the patient volume in the image. For each patient, the fractional weekly CBCT images are regarded as the reference images and the pCT image is rigidly registered to each $\mathrm{CBCT}$ image via the $3 \mathrm{D}$ slicer. The resolution of the transformed $\mathrm{pCT}$ is resampled to the size of the weekly $\mathrm{CBCT}$ images after rigid registration. To reduce computational time and to save memory, all the pCT and CBCT images were down-sampled to $256 \times 256$ to feed DIR after rigid registration.

\subsection{DIR algorithms}

Seven intensity-based DIR algorithms implemented in an open source toolkit, i.e. the DIRART [17], were tested. These DIR algorithms includes four optical flow based methods: the Original Horn and Schunck (HSO), the Horn and Schunck with Issam's non-linear smoothness (HS_INLS), the combined Horn-Schunck and Lucas-Kanade (HSLK) and the iterative optical flow (IOF); one level-set based method: the original level set motion (OLSM); one demons based method: the double force demons (DFD), and one iterative method: the free form deformation (FFD).

\subsection{Similarity metrics}

The DIR output includes a deformed pCT image and the corresponding DVFs that define the point-topoint mapping between voxels in the moving and the reference image. The resulting DVFs are used to propagate the OAR contours on the $\mathrm{pCT}$ to the weekly CBCT coordinates, and quantitatively compared with those manually delineated ground truth OARs on the weekly CBCT.

To quantitatively evaluate the agreement between the deformed OARs and the reference ones, three similarity metrics were used: Dice similarity coefficient (DSC), percentage error (PE) and Hausdorff distance (HD). Given the ground truth reference region $A$ and the deformed region $B$, and their corresponding boundary point sets $\vec{A}=\left\{a_{1}, \ldots, a_{p}\right\}$ and $\vec{B}=\left\{b_{1}, \ldots, b_{q}\right\}$, the DSC is defined as: $D S C=$ 


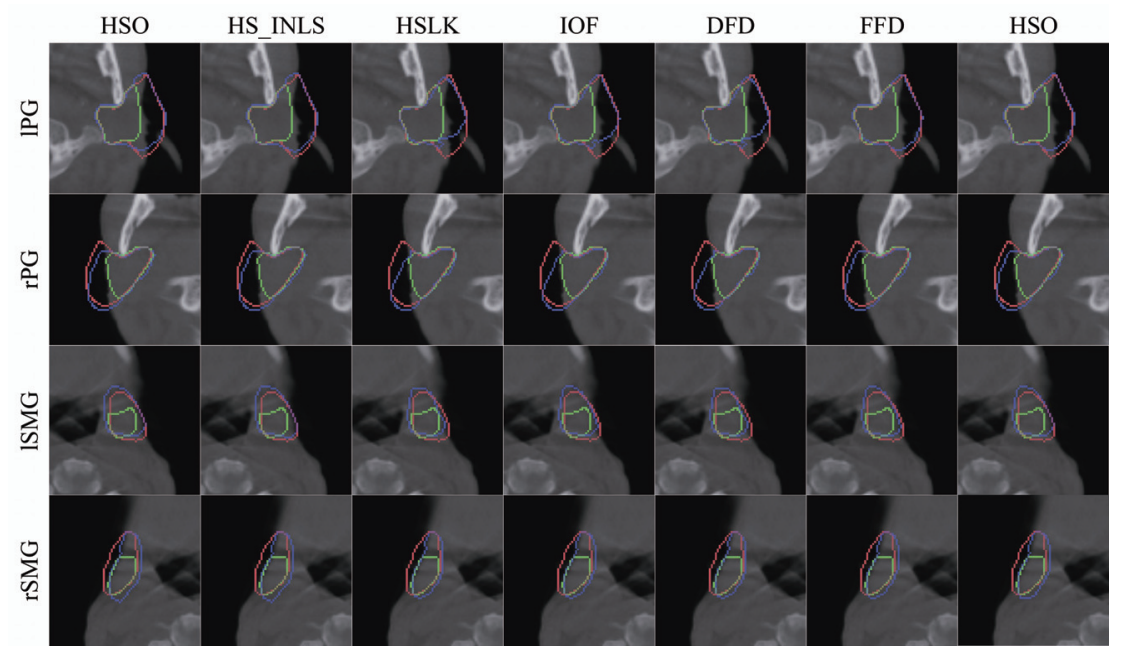

Fig. 1. Example OARs propagation results by the seven evaluated DIR algorithms. Columns: DIR algorithms; Rows: different OARs. The ground truth, rigid and deformable registration results are displayed in red, blue and green curves, respectively.

$2(A \cap B) /(A+B)$, which ranges from 0 to 1 , corresponding to the worst and the best segmentation, respectively. The PE is defined as: $P E=(A \cup B-A \bigcap B) /(A)$, with 0 representing the best segmentation. The HD is defined as: $H D=\max (h(\vec{A}, \vec{B}), h(\vec{B}, \vec{A}))$, where $h(\vec{A}, \vec{B})=\max _{a \in \vec{A}} \min _{b \in \vec{B}}\|a-b\|$ and $\|\cdot\|$ is the $\mathrm{L}_{2}$ norm on the points of $\vec{A}$ and $\vec{B}$.

\subsection{Data analysis}

Descriptive statistics was carried to characterize the volumetric changes of different OARs and variances in the weekly performance of each DIR algorithms over the 6-week treatment course. Independent Samples Kruskal-Wallis (K-W) test was performed to compare the DIR accuracy between different DIR algorithms. All statistical analyses were implemented with the SPSS 19.0 software (spss Inc., Chicago, IL), and the statistical significance was set at $p=0.05$.

\section{Results}

\subsection{Volume changes of OARs}

Volume decreases were found in both the PGs and SMGs (data not shown). At the end of the treatment, mean volume reductions of the $\mathrm{PPG}$ and $\mathrm{rPG}$ were $11.15 \pm 8.40 \mathrm{cc}$ and $8.03 \pm 6.55 \mathrm{cc}$, corresponding to a reduction of $29.66 \pm 19.04 \%$ and $22.89 \pm 18.21 \%$ related to the initial volumes on the pCT, respectively. The mean volume decrease rate of the $1 \mathrm{PG}$ was $1.65 \pm 1.17$ (4.45 $\pm 2.77 \%)$ per week, which is slightly higher than that of the rPG (1.48 $\pm 0.96 \mathrm{cc}$ per week, $4.04 \pm 2.63 \%$ per week).

As for the SMGs, the $1 \mathrm{SMGs}$ and rSMGs decreased by $10.88 \pm 14.45 \%(1.16 \pm 1.3 \mathrm{cc})$ and 13.87 $\pm 27.16 \%$ ( $1.54 \pm 1.85 \mathrm{cc})$, respectively, when compared with their initial planning volumes. In terms of the volume decrease rate, similar finding was observed: the ISMGs is greater than the rSMGs, with a decrease rate of $4.03 \pm 4.83 \%$ versus $3.55 \pm 4.13 \%$. 

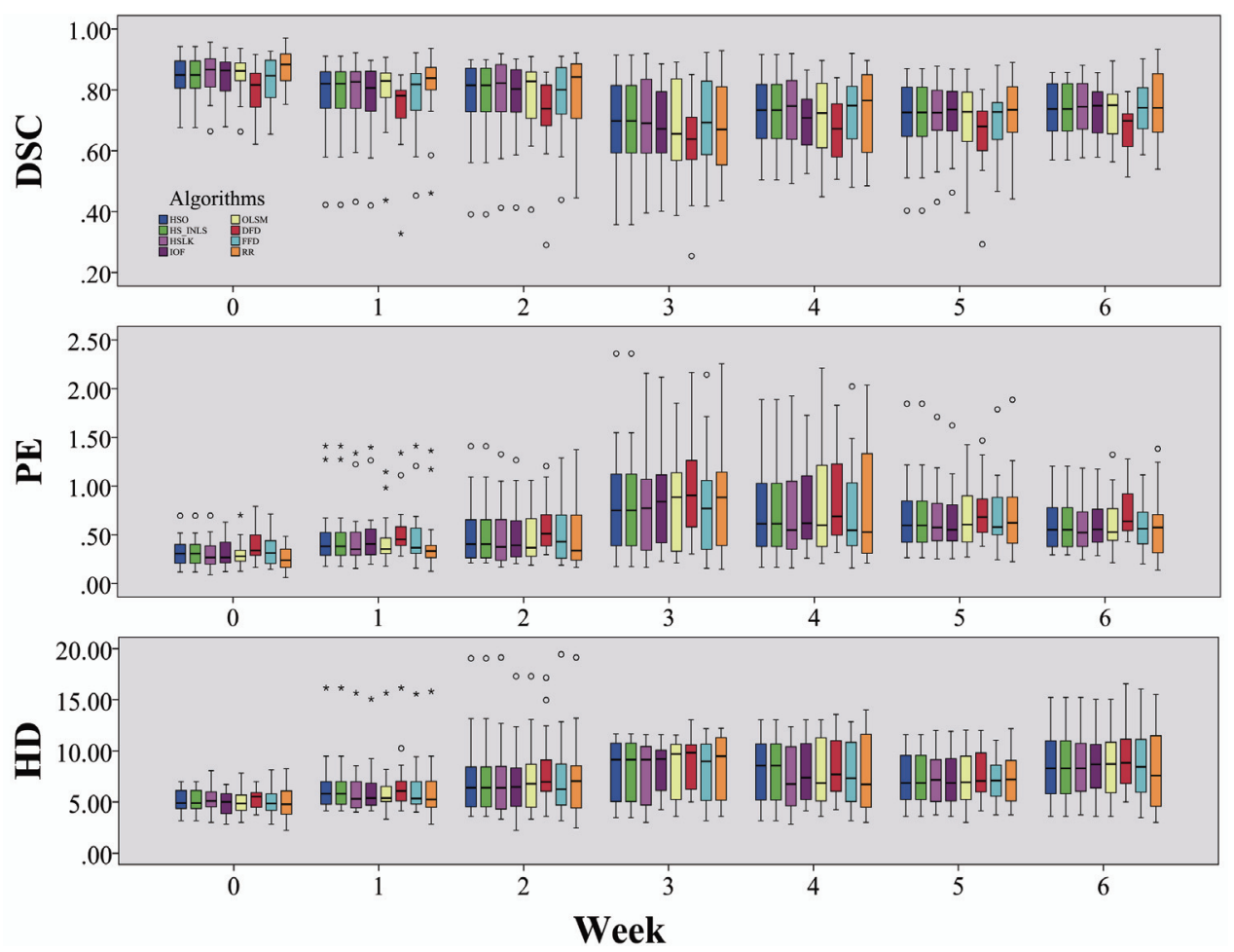

Fig. 2. Boxplots of the weekly DSC, PE and HD by different DIR algorithms. The limits of each box represent the 25th and 75th percentages, the whisker represents the minimum and maximum, and the bold black line represents the median value, and the circles represent outliers.

\subsection{General DIR performance over the treatment course}

Figure 1 showed an example (patient 1) of OARs propagation results by the seven evaluated DIR algorithms in the fourth week. And the weekly medians and inter-quartile ranges (IQRs) of the DSC, PE and HD for each DIR algorithm are showed in Fig. 2. We have the following observations: 1) the DFD performs worst among all methods over the treatment. However, the DFD is the most robust one since it has the shortest IQRs; 2 ) at the early stage of the treatment (Week 0 and 1 ), the rigid registration outperforms all the other DIR algorithms, and the HSLK performed best with median DSC $=0.867$ and $\mathrm{PE}=0.266 .3$ ) at the middle stage of treatment (Weeks 2, 3 and 4), both the rigid registration and all the DIR algorithms deteriorate, indicated by decreasing DSC and increasing PE. The OLSM and DFD performed even worse than the rigid registration in week 3. However, the DIR algorithms seem to be more robust than the rigid registration, proven by smaller IQRs. 4) at the end of treatment (Weeks 5 and 6), all the evaluated algorithms tended to be stable, and no evident discrepancy of DSC and PE was observed. 5) as for the HD, the time trend was similar to that of the other two metrics.

\subsection{DIR performance comparisons}

Figure 3 showed the comparison among all the algorithms. Compared with RR, no significant difference in DIR performance was found between different DIR algorithms across the entire treatment course except for DFD. The DFD performs significantly worse (mean \pm std, median; DSC: $0.705 \pm$ 

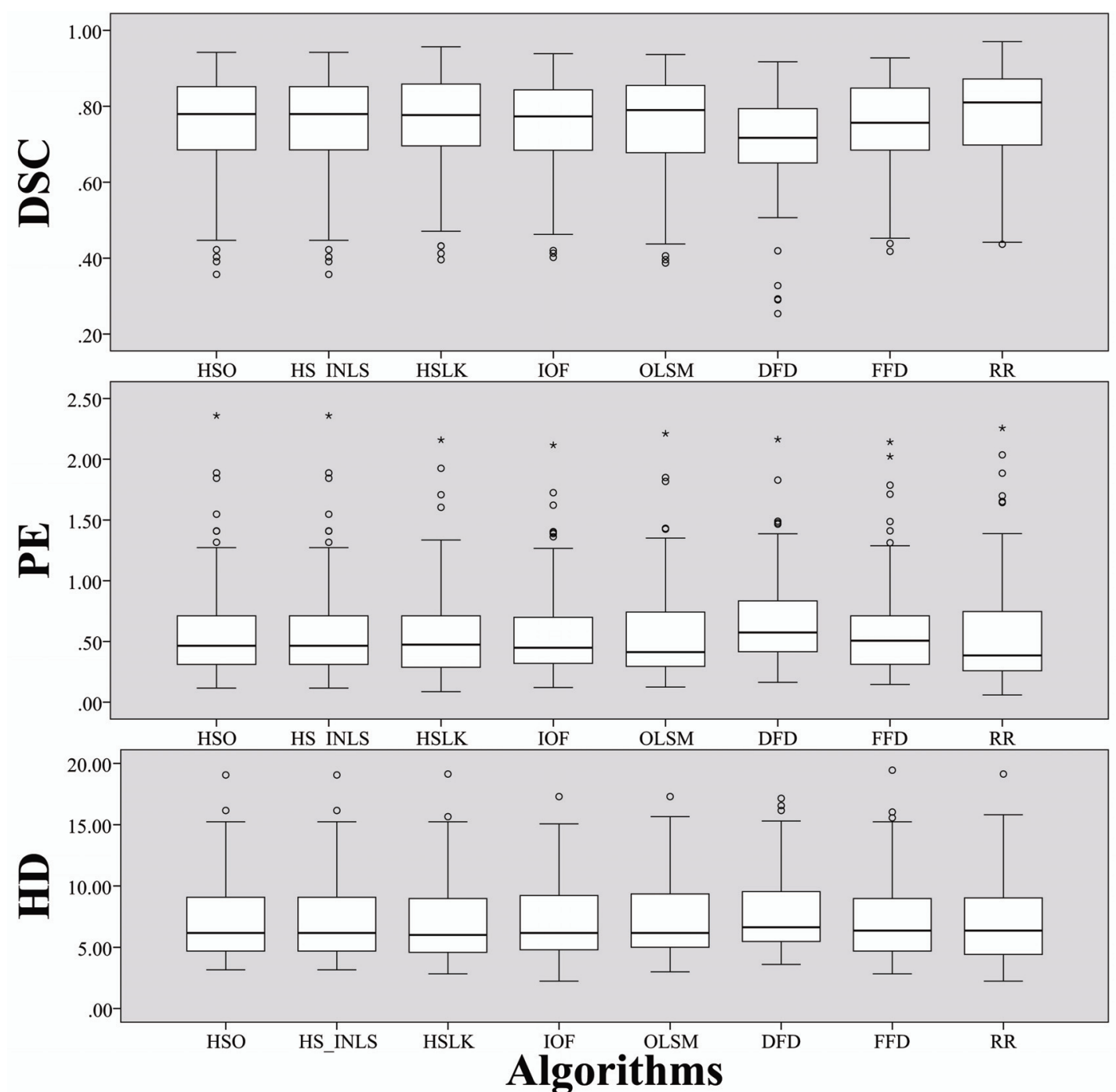

Fig. 3. Boxplots of DSC, PE and HD by different DIR algorithms for all OARs. The limits of each box represent the 25th and 75th percentages; the whisker represents the minimum and maximum, and the bold black line represents the median value. The asterisks and circles represent extremes and outliers.

$0.119,0.717$; PE: $0.658 \pm 0.343,0.573)$ than the RR (DSC $=0.773 \pm 0.130,0.810 ;$ PE: $0.559 \pm 0.431$, $0.385)$ (DSC: $p<0.01$; PE: $p<0.01)$. Surprisingly, the HSLK, which scores the highest DSC $(0.765$ $\pm 0.121,0.777)$ and lowest PE $(0.552 \pm 0.372,0.474)$ among all the evaluated DIR algorithms, was not better than RR. Followed by the HSLK are HSO $(0.758 \pm 0.121,0.780)$, HS_INLS $(0.758 \pm 0.121$, $0.780)$, OSLM $(0.758 \pm 0.122,0.790)$, FFD $(0.755 \pm 0.117,0.756)$ and IOF $(0.753 \pm 0.115,0.773)$. However, the difference between any two of those DIR algorithms was minor and negligible $(p>0.05)$. Again, the DSC of DFD was significantly lower than that of other DIRs $(p<0.01$ for all). Interestingly, the PE of DFD was significantly higher than that of only two DIRs, i.e. the HSLK $(p<0.01)$ and the $\operatorname{OLSM}(p=0.023)$. The IQR of DSC, which measures the consistency in DIR performance, for the DFD, HSO, HS_INLS, HSLK, IOF, OSLM and FFD were 0.143, 0.167, 0.167, 0.163, 0.160, 0.178 
and 0.165 , respectively. And similar situation occurs in the IQR of PE. The DFD and the OSLM score the lowest and highest IQR values, indicating the most and least robustness among all the evaluated approaches.

For similarity metric HD, DFD $(7.630 \pm 2.871)$ was apparently inferior to other DIRs, ranging from $7.019 \pm 3.297$ (RR) to $7.133 \pm 3.007$ (HSO and HS_INLS). However, no statistically significant difference was found among all the DIR methods $(p=0.273)$.

\section{Discussion and conclusion}

The purpose of this study was to develop a strict and objective methodology to compare different DIR strategies with their ability to propagate OAR contours between CT and CBCT image. Various intensitybased DIR algorithms, including HSO, HS_INLS, HSLK, IOF, OLSM, DFD and FFD were extensively validated employing planning $\mathrm{CT}$ and weekly $\mathrm{CBCT}$ images that retrospectively acquired from six NPC patients.

As for the volume change of OARs over the treatment course, we observed that the volume of $1 \mathrm{PG}$, rPG, ISMG and rSMG decrease by $29.66 \%, 22.89 \%, 10.88 \%$ and $13.87 \%$ at the end of the treatment, and the corresponding decrease rates per week are $4.45 \%, 4.04 \%, 4.03 \%$ and $3.55 \%$, respectively. These results were in accordance with those reported in previous literature . For instance, Barker Jr et al. [3] found that the median PGs volume loss was $28.1 \%$ and the median volume loss rate was $0.6 \%$ per day. Using weekly CT, Robar et al. [18] showed that the mean volume of IPG and rPG decreased by $4.4 \%$ and 5.0\% per week during a 6-week treatment course. Han et al. [19] and Lee et al. [20] observed a mean and median relative PGs loss rate of $1.1 \%$ and $0.7 \%$ per treatment day based on used daily megavoltage CT. Jin et al. [21] discovered that the left and right part of the PGs underwent a mean volume reduction of $4.4 \%$ and $4.7 \%$ per week based on weekly CBCT of ten NPC patients treated with IMRT, and the result was in agreement with the findings in Robar et al. [18] and ours. All these researches suggested that the OARs have considerable anatomical changes over the treatment course in $\mathrm{H} \& \mathrm{~N}$ patients. One interesting finding in this study is that the evaluated DIR algorithms did not outperform the rigid registration, which is different from published results that obtained from CT-CT DIR. For instance, Castadot et al. [8] compared twelve intensity-based DIR algorithms using CT images of five H \& N cancer patient and discovered that all the DIR strategies were significantly better than rigid registration based on the DSC metric. This conclusion was supported by the study of Rigaud et al. [22]. Interestingly, the demons based DIR algorithms scores the highest DSC in Castadot's and Rigaud's studies (DSC = 0.86 and 0.91 , respectively), in contrast, the demons based DFD algorithm performs worst in our study $(\mathrm{DSC}=0.71$ ). This significant discrepancy may be caused by different image quality between CT and CBCT. As pointed out by previous research [11], the image quality of a CBCT was inferior to that of the $\mathrm{CT}$ due to different image reconstruction algorithms, scatter artifacts, motion artifacts, etc. However, the results revealed in this study (mean DSC ranging from 7.05 to 7.65 ) are comparable with other studies on CT-CBCT DIR. For example, Huger et al. [23] reported a mean DSC of 7.0 for SMG on ten H \& $\mathrm{N}$ patients based on a block matching DIR algorithm. With the similar DIR strategy, Veiga et al. [24] obtained a mean DSC of 0.79 for normal tissues. The similarity metric PE exhibits similar properties as the DSC. In addition, the IQR of each DIR algorithm was smaller than that of the rigid registration, indicating that DIR was a more robust in registration performance. As for $\mathrm{HD}$, neither rigid registration nor DIR methods yield satisfactory performance.

In this study, we comprehensively compared the ability of OAR contour propagation by different DIR strategies in the context of CT-CBCT DIR for $\mathrm{H} \& \mathrm{~N}$ cancer patients. No significant difference was 
observed between rigid registration and the evaluated DIR algorithms except for DFD, which per formed worst. These results suggested that special care should be taken when utilizing the intensity-based DIR algorithms involved in this study to deform OAR contours between CT and CBCT, especially for those organs with low contrast.

\section{Acknowledgments}

This work is supported in part by the National Natural Science Foundation of China (no 81428019 and no 81301940), the Guangdong Natural Science Foundation (2015A030313302) and the 2015 Pearl River S\&T Nova Program of Guangzhou (201506010096).

\section{References}

[1] G.N. Marta, V. Silva, H. de Andrade Carvalho, F.F. de Arruda, S.A. Hanna, R. Gadia, J.L. da Silva, S.F. Correa, C.E. Vita Abreu and R. Riera, Intensity-modulated radiation therapy for head and neck cancer: Systematic review and metaanalysis. Radiother Oncol. 110, 9 (2014).

[2] B.M. Beadle, K.P. Liao, L.S. Elting, T.A. Buchholz, K.K. Ang, A.S. Garden and B.A. Guadagnolo, Improved survival using intensity-modulated radiation therapy in head and neck cancers: A SEER-Medicare analysis. Cancer. 120, 702 (2014).

[3] J.L. Barker Jr, A.S. Garden, K.K. Ang, J.C. O’Daniel, H. Wang, L.E. Court, W.H. Morrison, D.I. Rosenthal, K.S.C. Chao, S.L. Tucker, R. Mohan and L. Dong, Quantification of volumetric and geometric changes occurring during fractionated radiotherapy for head-and-neck cancer using an integrated CT/linear accelerator system. International Journal of Radiation Oncology Biology Physics. 59, 960 (2004).

[4] D. Yan, F. Vicini, J. Wong and A. Martinez, Adaptive radiation therapy. Phys. Med. Biol. 42, 123 (1997).

[5] D.L. Schwartz, A.S. Garden, S.J. Shah, G. Chronowski, S. Sejpal, D.I. Rosenthal, Y. Chen, Y. Zhang, L. Zhang, P.F. Wong, J.A. Garcia, K. Kian Ang and L. Dong, Adaptive radiotherapy for head and neck cancer-dosimetric results from a prospective clinical trial. Radiotherapy and Oncology: Journal of the European Society for Therapeutic Radiology and Oncology. 106, 80 (2013).

[6] M. Thor, J.B. Petersen, L. Bentzen, M. Hoyer and L.P. Muren, Deformable image registration for contour propagation from CT to cone-beam CT scans in radiotherapy of prostate cancer. Acta Oncol. 50,918 (2011).

[7] N. Wen, C. Glide-Hurst, T. Nurushev, L. Xing, J. Kim, H. Zhong, D. Liu, M. Liu, J. Burmeister, B. Movsas and I.J. Chetty, Evaluation of the deformation and corresponding dosimetric implications in prostate cancer treatment. Physics in Medicine and Biology. 57, 5361 (2012).

[8] P. Castadot, J.A. Lee, A. Parraga, X. Geets, B. Macq and V. Gregoire, Comparison of 12 deformable registration strategies in adaptive radiation therapy for the treatment of head and neck tumors. Radiotherapy and Oncology: Journal of the European Society for Therapeutic Radiology and Oncology. 89, 1 (2008).

[9] N. Hardcastle, W.A. Tome, D.M. Cannon, C.L. Brouwer, P.W. Wittendorp, N. Dogan, M. Guckenberger, S. Allaire, Y. Mallya, P. Kumar, M. Oechsner, A. Richter, S. Song, M. Myers, B. Polat and K. Bzdusek, A multi-institution evaluation of deformable image registration algorithms for automatic organ delineation in adaptive head and neck radiotherapy. Radiat. Oncol. 7, 90 (2012).

[10] A.S. Mohamed, M.N. Ruangskul, M.J. Awan, C.A. Baron, J. Kalpathy-Cramer, R. Castillo, E. Castillo, T.M. Guerrero, E. Kocak-Uzel, J. Yang, L.E. Court, M.E. Kantor, G.B. Gunn, R.R. Colen, S.J. Frank, A.S. Garden, D.I. Rosenthal and C.D. Fuller, Quality assurance assessment of diagnostic and radiation therapy-simulation CT image registration for head and neck radiation therapy: Anatomic region of interest-based comparison of rigid and deformable algorithms. Radiology. 274, 752 (2015).

[11] J. Hou, M. Guerrero, W. Chen and W.D. D'Souza, Deformable planning CT to cone-beam CT image registration in head-and-neck cancer. Medical Physics. 38, 2088 (2011).

[12] X. Zhen, X. Gu, H. Yan, L. Zhou, X. Jia and S.B. Jiang, CT to cone-beam CT deformable registration with simultaneous intensity correction. Phys. Med. Biol. 57, 6807 (2012).

[13] Y. Lou, T. Niu, X. Jia, P.A. Vela, L. Zhu and A.R. Tannenbaum, Joint CT/CBCT deformable registration and CBCT enhancement for cancer radiotherapy. Med. Image Anal. 17, 387 (2013).

[14] S. Nithiananthan, K.K. Brock, M.J. Daly, H. Chan, J.C. Irish and J.H. Siewerdsen, Demons deformable registration for CBCT-guided procedures in the head and neck: convergence and accuracy. Medical Physics. 36, 4755 (2009). 
[15] N. Hardcastle, W. van Elmpt, D. De Ruysscher, K. Bzdusek and W.A. Tome, Accuracy of deformable image registration for contour propagation in adaptive lung radiotherapy. Radiat. Oncol. 8, 243 (2013).

[16] A. Fedorov, R. Beichel, J. Kalpathy-Cramer, J. Finet, J.C. Fillion-Robin, S. Pujol, C. Bauer, D. Jennings, F. Fennessy, M. Sonka, J. Buatti, S. Aylward, J.V. Miller, S. Pieper and R. Kikinis, 3D Slicer as an image computing platform for the quantitative imaging network. Magn. Reson. Imaging. 30, 1323 (2012).

[17] D. Yang, S. Brame, I. El Naqa, A. Aditya, Y. Wu, S.M. Goddu, S. Mutic, J.O. Deasy and D.A. Low, Technical note: DIRART-A software suite for deformable image registration and adaptive radiotherapy research. Medical Physics. 38, 67 (2011).

[18] J.L. Robar, A. Day, J. Clancey, R. Kelly, M. Yewondwossen, H. Hollenhorst, M. Rajaraman and D. Wilke, Spatial and dosimetric variability of organs at risk in head-and-neck intensity-modulated radiotherapy. International Journal of Radiation Oncology, Biology, Physics. 68, 1121 (2007).

[19] C. Han, Y.J. Chen, A. Liu, T.E. Schultheiss and J.Y. Wong, Actual dose variation of parotid glands and spinal cord for nasopharyngeal cancer patients during radiotherapy. International Journal of Radiation Oncology, Biology, Physics. 70, 1256 (2008).

[20] C. Lee, K.M. Langen, W. Lu, J. Haimerl, E. Schnarr, K.J. Ruchala, G.H. Olivera, S.L. Meeks, P.A. Kupelian, T.D. Shellenberger and R.R. Manon, Evaluation of geometric changes of parotid glands during head and neck cancer radiotherapy using daily MVCT and automatic deformable registration. Radiotherapy and Oncology: Journal of the European Society for Therapeutic Radiology and Oncology. 89, 81 (2008).

[21] X. Jin, W. Hu, H. Shang, C. Han, J. Yi, Y. Zhou and C. Xie, CBCT-based volumetric and dosimetric variation evaluation of volumetric modulated arc radiotherapy in the treatment of nasopharyngeal cancer patients. Radiat Oncol. 8, 279 (2013).

[22] B. Rigaud, A. Simon, J. Castelli, M. Gobeli, J.D. Ospina Arango, G. Cazoulat, O. Henry, P. Haigron and R. De Crevoisier, Evaluation of deformable image registration methods for dose monitoring in head and neck radiotherapy. BioMed Research International. 2015, 726268 (2015).

[23] S. Huger, P. Graff, V. Harter, V. Marchesi, P. Royer, J.C. Diaz, S. Aouadi, D. Wolf, D. Peiffert and A. Noel, Evaluation of the block matching deformable registration algorithm in the field of head-and-neck adaptive radiotherapy. Physica Medica: An International Journal Devoted to the Applications of Physics to Medicine and Biology: Official Journal of the Italian Association of Biomedical Physics. 30, 301 (2014).

[24] C. Veiga, J. McClelland, S. Moinuddin, A. Lourenco, K. Ricketts, J. Annkah, M. Modat, S. Ourselin, D. D’Souza and G. Royle, Toward adaptive radiotherapy for head and neck patients: Feasibility study on using CT-to-CBCT deformable registration for "dose of the day" calculations. Medical Physics 41, 031703 (2014). 